\section{Funcionamiento cognitivo en adultos mayores: rol de la reserva cognitiva, apoyo social y depresión}

\author{
MARGARITA CANCINO ${ }^{1, a}$, LUCIO REHBEIN FELMER ${ }^{1, b}$, \\ MANUEL S. ORTIZ ${ }^{1,2, c}$
}

\section{Cognitive reserve, depression and social support. Analysis of 206 older adults}

Background: Cognitive reserve (CR) is a protective factor in aging. Depression and perceived social support are associated with cognitive performance in older adults. However, their role in the relationship between CR and cognitive functioning is less clear. Aim: To determine the relationship between CR and cognitive functioning and whether this relationship is mediated by depression and moderated by social support. Material and Methods: $C R$, depression, perceived social support, and cognitive functioning scales were applied to a convenience sample of 206 older adults, aged $69 \pm 1$ years (77\% women). Structural equation analysis and moderate mediation analysis were performed. Results: There was a direct effect of $C R$ in cognitive functioning $(\beta=0.223, p=0.005)$, which was not mediated by depression ( $\beta=0.040, p=0.096)$. High $C R$ scores were associated with lower depression scores $(\beta=-0.203, p=0.002)$. Higher depression scores were associated with worse cognitive functioning $(\beta=-0.168, p=0.040)$. The effect of CR on depression was moderated by social support $(\beta=-0.161, p=$ $0.032)$ controlling for income and age. Conclusions: The relationship between $C R$ and cognition in older adults allows an early assessment of cognitive dysfunction risk. Depression is an independent risk factor for cognitive functioning. Social support protects individuals with high CR from developing depression.

(Rev Med Chile 2018; 146: 315-322)

Key words: Aged; Cognitive Reserve; Depression; Social Support.

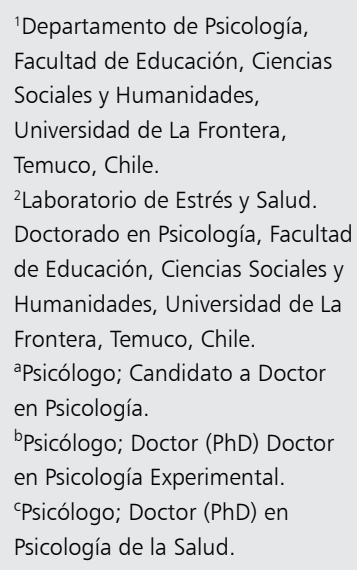

Esta investigación fue financiada por CONICYT (Comisión Nacional de Investigación Científica y Tecnológica, Gobierno de Chile), Gastos operacionales Doctorado Nacional № Folio 21140543.

Recibido el 21 de julio de 2017 , aceptado el 23 de marzo de 2018.

Correspondencia a: Margarita Cancino, Departamento de Psicología, Universidad de La Frontera. Montevideo 0830, Temuco, Chile.

Teléfono: 56452732443 margarita.cancino@ufrontera.cl
L a expectativa de vida a nivel global ha tenido un aumento sostenido en el tiempo. En Chile, la expectativa de vida para las mujeres alcanza los 85 años y para los hombres los 80 años ${ }^{1}$. El creciente envejecimiento poblacional exige la planificación y puesta en marcha de planes de salud pública que contribuyan al envejecimiento activo ${ }^{1}$. La evidencia indica que con la edad aumenta considerablemente el riesgo de enfermedad y dependencia, en consecuencia, 23\% del gasto en salud a nivel mundial está reservado para la atención de adultos mayores de 60 años². Un estudio realizado en Chile para evaluar la atención geriátrica reportó que las patologías más prevalentes fueron hipertensión arterial, obesidad, diabetes mellitus, depresión y demencia ${ }^{3}$. Para esta última se ha identificado como un factor de riesgo la presencia de deterioro cognitivo, el cual se ha definido como la alteración de una o más funciones cognitivas, pudiendo afectar también el funcionamiento en la vida cotidiana ${ }^{4}$.

Los estudios del funcionamiento cognitivo en los adultos mayores se han orientado a medir diferentes procesos cognitivos, especialmente memoria, atención, orientación, habilidades visoespaciales y lenguaje $e^{5-8}$, incluyendo, además, su rol 
en el envejecimiento activo. Una de las variables que más atención ha recibido en los últimos años es la reserva cognitiva (RC), definida como la forma eficiente y flexible en que un sujeto es capaz de optimizar su rendimiento cognitivo y responder a las demandas del entorno ${ }^{9,10}$. La capacidad de respuesta del sujeto es explicada por la forma en que una eficiente red neuronal es utilizada y modulada en el envejecimiento normal, pero también en presencia de patología ${ }^{11,12}$. La RC se acumula a lo largo de la vida e incluye elementos innatos y adquiridos $^{13}$, como, por ejemplo, la escolaridad, tipo de ocupación y la realización de actividades cognitivamente desafiantes y de ocio en la adultez y la adultez mayor ${ }^{14-16}$. Los sujetos con una alta RC tienen menor riesgo de ser diagnosticados con demencia, o presentar una progresión más lenta de esta ${ }^{11-19}$.

Otra variable que ha recibido creciente atención en relación al funcionamiento cognitivo es la depresión. Al respecto, la evidencia teórica y empírica es divergente; por una parte se sostiene que la presencia de sintomatología depresiva es un factor de riesgo para el desarrollo de deterioro cognitivo y la posterior conversión a demencia, especialmente la de tipo Alzheimer y vascular ${ }^{20-22}$, por lo tanto, esta tendría un efecto en el funcionamiento cognitivo, principalmente cuando la depresión se presenta tardíamente en el ciclo vital, aumentando considerablemente el riesgo de demencia de tipo vascular ${ }^{20}, y$, por otra parte, se plantea que la disminución en el rendimiento cognitivo podría ser explicada por la presencia de sintomatología depresiva aguda, especialmente por la reducción en los tiempos de reacción, memoria y funcionamiento ejecutivo, originando perfiles heterogéneos de declive en los diferentes dominios cognitivos ${ }^{23}$. Adicionalmente, un estudio reciente reportó que los síntomas depresivos moderan la relación entre RC y cognición, concluyendo que los adultos mayores que alcanzaron mayores niveles de RC tuvieron un menor rendimiento cognitivo en la medida que los síntomas depresivos aumentaron ${ }^{24}$. La asociación entre depresión y cognición ha sido probada en diferentes estudios ${ }^{25-27}$, destacando que los síntomas depresivos correlacionan con un rendimiento deficiente en lenguaje, memoria, función ejecutiva y atención ${ }^{28}$. De esta forma, es posible postular que la depresión puede ser una variable que medie la relación entre RC y funcionamiento cognitivo.
Si bien los síntomas depresivos y la depresión son un factor de riesgo para el deterioro cognitivo ${ }^{29}$ y diferentes tipos de demencia ${ }^{20,21,30}$, es sabido que el apoyo social es una variable que puede moderar los efectos negativos de la depresión y el estrés ${ }^{31}$; un estudio reporta que en presencia de altos niveles de apoyo social percibido, disminuyen los niveles de soledad percibida, lo cual se asocia con sintomatología depresiva. El apoyo social es un factor protector del curso del envejecimiento, los individuos que reciben más apoyo social experimentan menos declive del funcionamiento cognitivo ${ }^{32}$. Este efecto podría explicarse porque los adultos mayores que tienen una mayor integración y vínculos sociales desarrollan más y mejores estrategias de comunicación e interacción interpersonal, lo que reduce las consecuencias de la depresión y ejerce beneficios en el bienestar y la cognición ${ }^{33,34}$.

Considerando lo anterior, este estudio tuvo por objetivo determinar la relación que existe entre RC y funcionamiento cognitivo y si esta relación está mediada por la depresión y moderada por el apoyo social en adultos mayores de Temuco.

\section{Material y Método}

\section{Participantes}

Se seleccionó, por medio de un muestreo no probabilístico por conveniencia, una muestra de 206 adultos mayores chilenos, cuyo promedio de edad fue de 69 años (D.T. = 0,5). El 77\% de los participantes eran mujeres, $80 \%$ estaban jubilados; el ingreso promedio mensual era de \$369.213. La escolaridad promedio fue de 11,7 años (D.T. $=5,9$ ) Tabla 1. Los criterios de exclusión fueron presentar uno o más de los siguientes antecedentes: Lesión cerebral durante los últimos 5 años, diagnóstico de trastorno depresivo mayor o síndrome confusional en los últimos 6 meses.

\section{Instrumentos}

Funcionamiento cognitivo: Se utilizó la versión en español del Addenbrooke's Cognitive Examination revisado (ACE-R $)^{35}$. Es una prueba cognitiva breve que evalúa seis dominios cognitivos: orientación, atención, memoria, fluidez verbal, lenguaje y habilidades visoespaciales. La consistencia interna fue de 0,81 .

Depresión: Los sujetos contestaron la Escala de Depresión Geriátrica de Yesavage ${ }^{36}$, conformada 
Tabla 1. Antecedentes de salud del total de participantes

\begin{tabular}{|c|c|}
\hline Antecedentes de salud & $\%$ \\
\hline Hipertensión arterial & 54,85 \\
\hline Diabetes & 17,48 \\
\hline Hipercolesterolemia & 46,12 \\
\hline Consume alcohol & 66,5 \\
\hline Consume tabaco & 14,08 \\
\hline
\end{tabular}

por 15 ítems, con un formato de respuesta dicotómica (sí - no). El alfa ordinal del instrumento fue de 0,71 .

Apoyo social percibido: Se utilizó ENRICHD Social Support Inventory (ESSI) ${ }^{37}$. Consta de seis ítems que miden la frecuencia de situaciones ocurridas en el último mes. El formato de respuesta es una escala de frecuencia de cuatro puntos (0 = "nunca" a $4=$ "siempre"). La consistencia interna del instrumento fue de 0,73 .

Reserva cognitiva: Evaluada con la escala de Reserva Cognitiva ${ }^{38}$ la cual está compuesta por 24 ítems que indagan la frecuencia con que el sujeto realiza determinadas actividades de la vida diaria, de formación o acceso a información, etc. El formato de respuesta es una escala de frecuencia de cuatro puntos $(0=$ "nunca" a $4=$ "tres o más veces a la semana"). La consistencia interna del instrumento fue de 0,74 .

Covariables: Los sujetos autorreportaron su edad, años de escolaridad e ingreso mensual, presencia o ausencia de hipertensión, diabetes e hipercolesterolemia, y el uso de alcohol y tabaco.

\section{Procedimiento}

Los participantes fueron contactados en diferentes organizaciones para el adulto mayor en la ciudad de Temuco. Quienes aceptaron voluntariamente participar de este estudio firmaron un consentimiento informado. Los participantes fueron evaluados por el equipo de investigación en su domicilio o en dependencias de la Universidad de La Frontera según sus preferencias; en ambos espacios físicos se aseguró el cumplimiento de los estándares requeridos. La evaluación tuvo una duración promedio de 100 min y una vez finalizada fue retribuida con dos mil pesos. Este estudio
Tabla 2. Nivel de escolaridad del total de participantes

\begin{tabular}{|lr|}
\hline Nivel de escolaridad & $\mathbf{\%}$ \\
\hline Sin escolaridad & 1,5 \\
\hline Enseñanza Básica incompleta & 15,5 \\
\hline Enseñanza Básica completa & 11,2 \\
\hline Enseñanza Media incompleta & 13,6 \\
\hline Enseñanza Media completa & 20,3 \\
\hline Estudios de nivel técnico superior incompletos & 1,5 \\
\hline Estudios de nivel técnico superior completos & 7,8 \\
\hline Estudios universitarios incompletos & 5,3 \\
\hline Estudios universitarios completos & 14,6 \\
\hline Estudios de posgrado & 8,7 \\
\hline
\end{tabular}

fue autorizado por el Comité Ético Científico de la Universidad de La Frontera y cumple con los principios éticos de la declaración de Helsinki.

\section{Análisis de datos}

Se realizaron análisis descriptivos para caracterizar a los participantes del estudio (Tabla 2) y se efectuó el análisis factorial confirmatorio del instrumento para evaluar funcionamiento cognitivo y calcular las cargas factoriales de los ítems en cada proceso cognitivo. Finalmente, se realizaron análisis de ecuaciones estructurales y mediación moderada, considerándose indicadores de bondad de ajuste convencionales ${ }^{39}$ : chi-cuadrado no significativo, índices de ajuste comparativo CFI $\geq 0,95$ y TLI $\geq 0,90$, índices de ajuste basado en los residuos SRMR $\leq 0,08$ y RMSEA $\leq 0,06$. Como método de estimación se utilizó máxima verosimilitud. Todos los análisis fueron realizados con el software STATA 14.1

\section{Resultados}

Para identificar posibles diferencias en los promedios entre hombres y mujeres en las variables de interés del estudio, se realizó una análisis de prueba t de Student para muestras independientes, en donde solo se identificaron diferencias estadísticamente significativas en $\mathrm{RC}$, hombres con un promedio de $47,21(\mathrm{DT}=12,77)$ y mujeres 54,19 $(\mathrm{DT}=10,53), t(182)=-3,614 ; \mathrm{p}=0,001$. 
El análisis factorial confirmatorio del ACE-R demostró una estructura de un factor latente, denominado funcionamiento cognitivo, con cuatro indicadores, conformados por los procesos cognitivos que presentaron cargas factoriales superiores a 0,30 , estos procesos fueron: lenguaje, atención, memoria y habilidades visoespaciales (Figura 1). Se excluyeron del modelo los procesos de orientación y fluidez verbal, dado que presentaron bajas cargas factoriales.

Para probar el efecto directo de la RC en el funcionamiento cognitivo y la mediación de esta relación a través de la depresión, se ejecutó un modelo de ecuaciones estructurales. Respecto a los efectos directos, la RC tuvo un efecto en la depresión $(\beta=-0,203, p=0,002)$ y en el funcionamiento cognitivo $(\beta=0,223, p=0,005)$, mientras que la depresión tuvo un efecto directo en el funcionamiento cognitivo $(\beta=-0,168, p=0,040)$; respecto a los efectos indirectos, no existió una mediación a través de la depresión: $\beta=0,040, p=0,096$, sin embargo, el efecto total del modelo fue estadísticamente significativo: $\beta=0,306, p=0,004$.
Al incluir el apoyo social percibido como moderador de la relación entre RC y depresión, el efecto directo entre ambas variables dejó de ser estadísticamente significativo $(\beta=-0,130, p=0,085)$, sin embargo, se observó que el apoyo social percibido tenía un rol moderador $(\beta=-0,161, p=0,032)$ en esta relación, tal que a mayores niveles de apoyo social percibido y mayores niveles de RC, menor fue el nivel de depresión. Respecto al efecto directo entre depresión y funcionamiento cognitivo, al controlar por renta y edad, este efecto dejó de ser significativo $(\beta=-0,111, p=0,178)$. El efecto de la renta $(\beta=0,283, \mathrm{p}=0,000)$ y la edad ( $\beta=-0,213, p=0,008)$ en el funcionamiento cognitivo fueron significativos. El efecto de la RC en el funcionamiento cognitivo, se mantuvo estadísticamente significativo $(\beta=0,154, p=0,057)$, luego de incluir estas covariables. El ajuste final del modelo, incluyendo la mediación moderada, tuvo excelentes indicadores de bondad de ajuste: $\chi_{(\mathrm{gl}=11)}^{2}=16,787, \mathrm{p}=0,114$, RMSEA $=0,051(0,000$; $0,097), \mathrm{SRMR}=0,042, \mathrm{CFI}=0,966, \mathrm{TLI}=0,939$ y CD $=0,109$ (Figura 2).

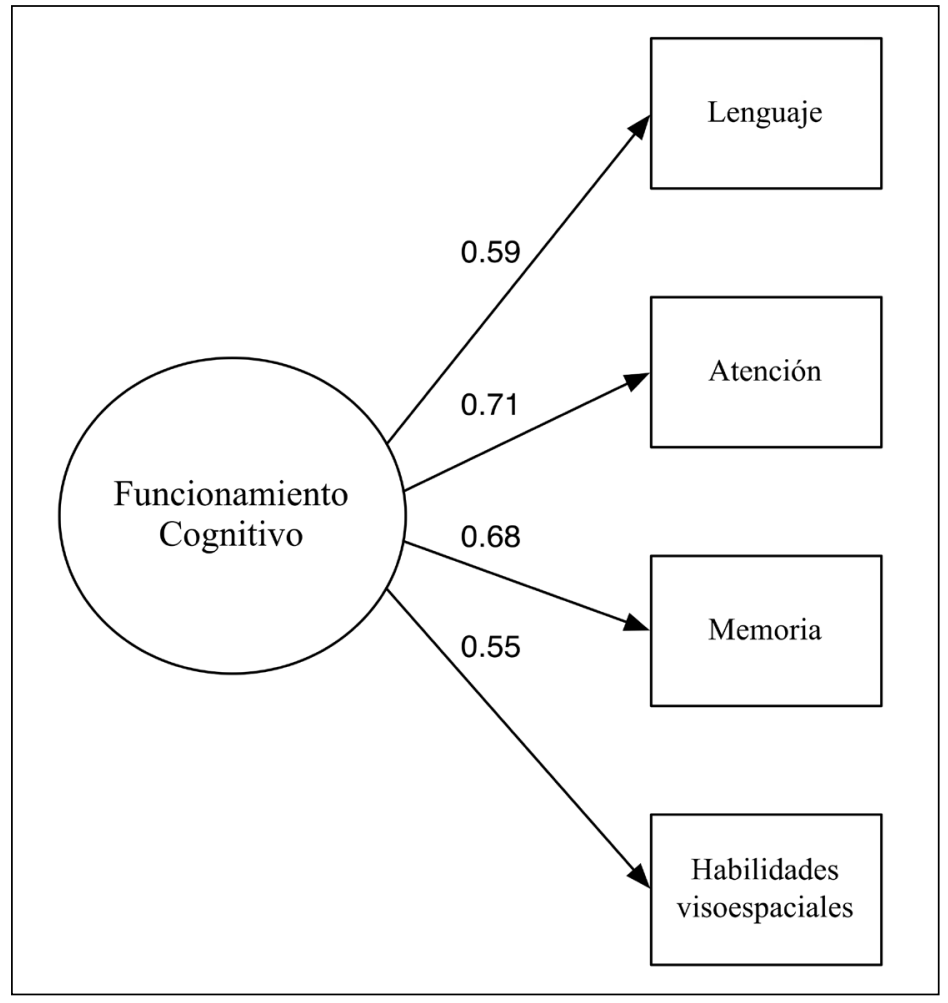

Figura 1. Estructura factorial del Addenbrooke's Cognitive Examination (ACE-R) y las cargas factoriales de cada proceso cognitivo. 


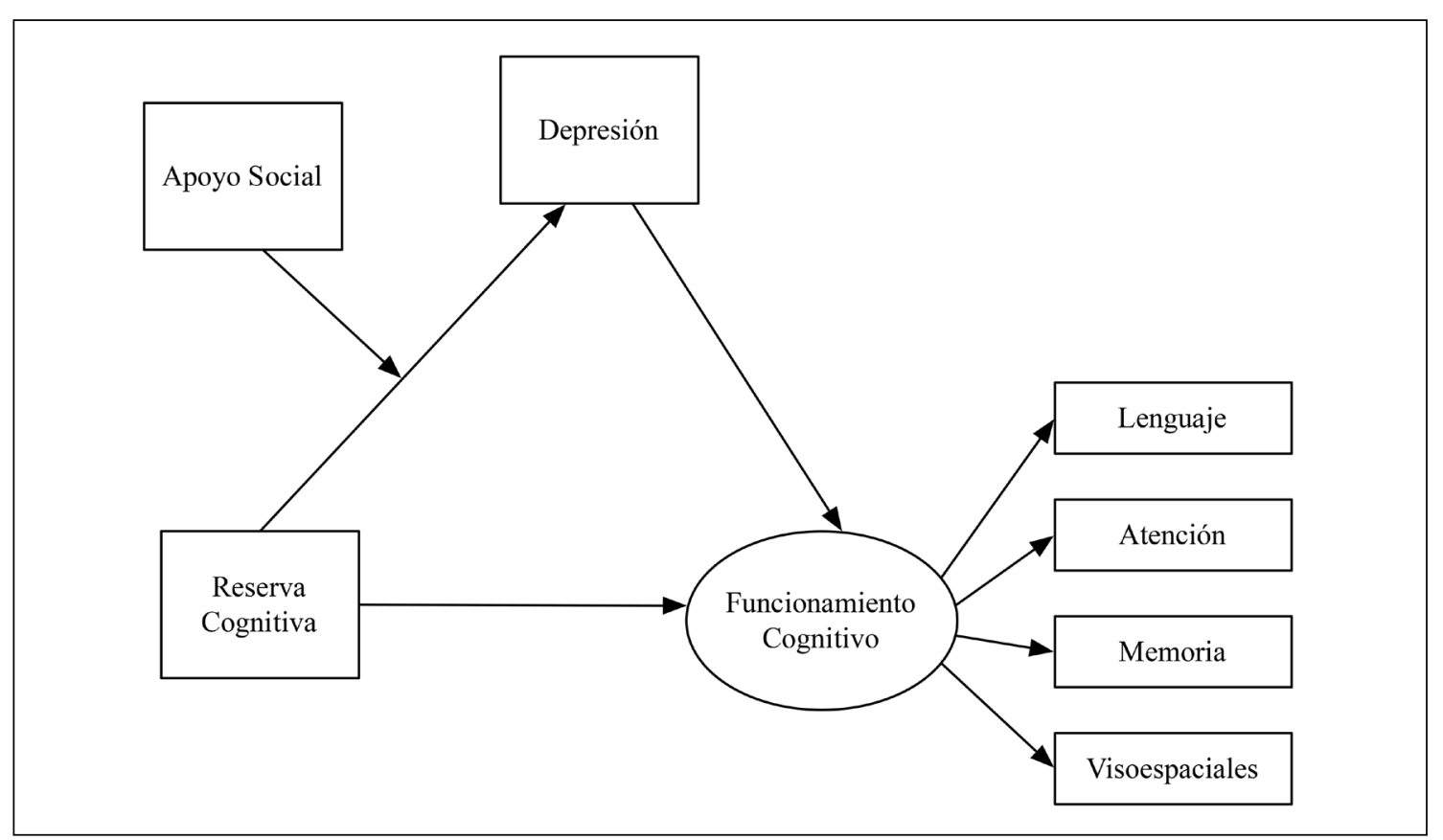

Figura 2. Modelo de relaciones de las variables en estudio. $\chi^{2}(g l=11)=16.787, p=0,114, S R M R=0,042, R M S E A=0,051$ $(0,000 ; 0,097), C F I=0,966, T L I=0,939$ y $C D=0,109$.

\section{Discusión}

El objetivo de este estudio fue determinar la relación que existe entre la $\mathrm{RC}$ y el funcionamiento cognitivo y el rol de la depresión y el apoyo social. Los resultados obtenidos evidencian un efecto directo de la RC en el funcionamiento cognitivo, lo cual corrobora los beneficios cognitivos y funcionales que la $\mathrm{RC}$ tiene en el rendimiento de los adultos mayores. Este efecto es robusto, puesto que al controlar por depresión, edad y renta, este efecto directo en el desempeño cognitivo de los sujetos se mantiene inalterable. De esta forma, la RC emerge como un componente protector activo de los recursos que un sujeto despliega para resolver satisfactoriamente tareas que demanden la activación de procesos cognitivos, tales como lenguaje, atención, memoria y habilidades visoespaciales, ya que una alta $\mathrm{RC}$ se traduce en conexiones neuronales más flexibles y eficaces, las que permiten al sujeto hacer frente con éxito a una patología neurodegenerativa ${ }^{11,40} \mathrm{o}$ lograr un envejecimiento activo.

Con respecto al rol de la depresión, los hallazgos de este estudio permiten confirmar una relación directa entre la depresión y el funcionamiento cognitivo, evidenciándose que la presencia de depresión en el adulto mayor es un factor de riesgo independiente para el eventual desarrollo de deterioro cognitivo, en este sentido, se hace esencial que el control médico y de enfermería que reciben los adultos mayores incorpore un control preventivo que permita pesquisar los primeros síntomas de depresión, con el objetivo de detectar tempranamente el riesgo potencial de interferir con el óptimo funcionamiento cognitivo de los sujetos envejecidos.

En cuanto al rol mediador de la variable depresión en la relación entre la RC y funcionamiento cognitivo, es posible que en este estudio no se alcanzara la suficiente potencia estadística para detectar la mediación, ya que el tamaño del efecto fue muy pequeño, sin embargo, existe una relación significativa entre depresión y funcionamiento cognitivo, por lo tanto, debe ser una variable a considerar en estudios con población envejecida y en los planes y programas de salud dirigidos a este grupo.

Respecto al apoyo social, esta investigación aporta evidencia empírica del rol moderador que 
tiene sobre la relación entre $\mathrm{RC}$ y depresión, es decir, sujetos con altos niveles de RC y alto apoyo social percibido experimentan menos depresión, lo que disminuye el riesgo de disfunción en los diferentes dominios cognitivos evaluados. Por otra parte, el apoyo social favorece el estado de salud y la percepción de seguridad de los adultos mayores, disminuyendo los sentimientos de soledad que sobrevienen con los años ${ }^{41}$, especialmente en aquellos adultos mayores que han enviudado y cuyos vínculos sociales se ven mermados por la muerte y la lejanía de familiares y amigos.

En este estudio se consideraron dos covariables (edad y renta), ya que diferentes investigaciones de envejecimiento y cognición reportan que la edad tiene un influjo en el rendimiento $\operatorname{cognitivo~}^{42-45}$, especialmente en la memoria, la función ejecutiva, la velocidad del procesamiento de información y el pensamiento. En la medida que aumenta la edad, los sujetos disminuyen su rendimiento cognitivo, pues la inserción de esta variable suprime los efectos que la depresión tiene en el funcionamiento cognitivo. Lo mismo ocurre con renta, la cual se asocia con un mejor estado de salud y tiene un impacto en diferentes dimensiones de la vida ${ }^{46}$.

Esta investigación tiene las limitaciones propias de un estudio transversal, no siendo posible identificar, por ejemplo, el rol de la RC en las trayectorias del funcionamiento cognitivo de los adultos mayores. Pese a lo anterior, no existen otros estudios reportados con población chilena que hayan probado empíricamente el efecto directo de la RC en el funcionamiento cognitivo. Otra limitación es que $77 \%$ de la muestra son mujeres y existe evidencia de que hombres y mujeres han tenido un acceso distinto a la educación y la ocupación ${ }^{47}$, por lo tanto, es posible inferir que algunos componentes de la RC son también distintos para hombres y mujeres, pues una parte importante de la RC recibe el influjo de la educación y el tipo de ocupación. Será importante que en próximos estudios sea posible contar con una muestra más equitativa en la variable sexo, ya que una parte importante de los estudios que evalúan capacidades cognitivas en el adulto mayor tributan al diagnóstico temprano y es muy importante identificar diferencias de sexo, ya sea para elaborar un diagnóstico o intervenciones más precisas ${ }^{48}$.

La realización de este estudio permitió indagar el rol de la RC en el envejecimiento, el efecto del apoyo social y los riesgos de la presencia de depresión, por lo tanto, es posible señalar que la evaluación cognitiva temprana, considerando el influjo de la edad y el estado afectivo de un sujeto, permitirán determinar tempranamente el riesgo de deterioro cognitivo y evaluar su eventual conversión a la demencia ${ }^{4}$, especialmente en un país que transita aceleradamente hacia el envejecimiento poblacional, estimándose que en el año 2050 los adultos mayores de 60 años constituirán $30 \%$ de la población total ${ }^{1}$.

De acuerdo a lo anterior, el interés por desarrollar estudios en adultos mayores es comprensible e invita a desarrollar futuras líneas de investigación que expliquen las claves de prevención de la disfunción cognitiva en el envejecimiento. En este contexto, el desarrollo de estudios longitudinales contribuiría al objetivo de identificar qué elementos de la RC favorecen a determinados dominios cognitivos y cómo en función de la RC se registran perfiles heterogéneos de declive cognitivo, pero también de envejecimiento activo por medio de la compensación cognitiva y funcional.

Agradecimientos: Este estudio recibió el apoyo de la Comisión Nacional de Investigación Científica y Tecnológica (CONICYT) del Gobierno de Chile, a través del Programa de Formación de Capital Humano Avanzado, Beca de Doctorado Nacional y Gastos operacionales 2014/ 21140543. Los autores agradecen profundamente a la Dirección de la Clínica Psicológica de la Universidad de La Frontera por facilitar el espacio físico para la recolección de los datos, a las diferentes agrupaciones del adulto mayor y a cada uno de los participantes de este estudio.

\section{Referencias}

1. Organización Mundial de la Salud. Informe Mundial Sobre El Envejecimiento Y La Salud.; 2015.

2. Prince MJ, Wu F, Guo Y, Gutiérrez Robledo LM, O'Donnell M, Sullivan R, et al. The burden of disease in older people and implications for health policy and practice. Lancet 2015; 385 (9967): 549-62.

3. Díaz V, Díaz I, Rojas G, Novogrodsky D. Evaluación geriátrica en la atención primaria. Rev Med Chile 2003; 131(8): 895-901.

4. Cancino M, Rehbein L. Factores de riesgo y precursores del Deterioro Cognitivo Leve (DCL): Una mirada sinóptica. Ter Psicológica 2016; 34 (3): 183-9. 
5. Nyberg L, Lövdén M, Riklund K, Lindenberger U, Bäckman L. Memory aging and brain maintenance. Trends Cogn Sci 2012; 16 (5): 292-305.

6. Saunders NLJ, Summers MJ. Attention and working memory deficits in mild cognitive impairment. J Clin Exp Neuropsychol 2010; 32 (4): 350-7.

7. Brum PS, Forlenza OV, Yassuda MS. Cognitive training in older adults with mild cognitive impairment: Impact on cognitive and functional performance. Dement Neuropsychol 2009; 3 (2): 124-31.

8. Juncos-Rabadán O, Pereiro AX, Facal D, Reboredo A, Lojo-Seoane C. Do the Cambridge Neuropsychological Test Automated Battery episodic memory measures discriminate amnestic mild cognitive impairment? Int J Geriatr Psychiatry 2014; 29 (6): 602-9.

9. Scarmeas N, Stern Y. Cognitive Reserve and Lifestyle. J Clin Exp Neuropsychol 2003; 25 (5): 625-33.

10. Stern Y. Cognitive reserve in ageing and Alzheimer's disease. Lancet Neurol 2012; 11 (11): 1006-12.

11. Barulli D, Stern Y. Efficiency, capacity, compensation, maintenance, plasticity: Emerging concepts in cognitive reserve. Trends Cogn Sci 2013; 17 (10): 502-9.

12. Colangeli S, Boccia M, Verde P, Guariglia P, Bianchini F, Piccardi L. Cognitive Reserve in Healthy Aging and Alzheimer's Disease. Am J Alzheimer's Dis Other Dementias ${ }^{\circledR} 2016$; 31 (5): 443-9.

13. Stern Y. Cognitive reserve. Neuropsychologia. 2009; 47 (10): 2015-28.

14. Tucker AM, Stern Y. Cognitive reserve in aging. Curr Alzheimer Res 2011; 8 (4): 354-60.

15. Wu Y-T, Teale J, Matthews FE, Brayne C, Woods B, Clare L. Lifestyle factors, cognitive reserve, and cognitive function: results from the Cognitive Function and Ageing Study Wales, a population-based cohort. Lancet 2016; 388: S114.

16. Clare L, Wu Y-T, Teale JC, MacLeod C, Matthews F, Brayne C, et al. Potentially modifiable lifestyle factors, cognitive reserve, and cognitive function in later life: A cross-sectional study. PLOS Med 2017; 14 (3): 1-14.

17. Meng X, D'Arcy C. Education and dementia in the context of the cognitive reserve hypothesis: A systematic review with meta-analyses and qualitative analyses. PLoS One 2012; 7 (6): 1-16.

18. Lo RY, Jagust WJ. Effect of cognitive reserve markers on Alzheimer pathologic progression. Alzheimer Dis Assoc Disord 2013; 27 (4): 343-50.

19. Hindle JV, Hurt CS, Burn DJ, Brown RG, Samuel M, Wilson KC, et al. The effects of cognitive reserve and lifestyle on cognition and dementia in Parkinson's disease-a longitudinal cohort study. Int J Geriatr Psychiatry 2016; 31 (1): 13-23.
20. Diniz BS, Butters MA, Albert SM, Dew MA, Reynolds CF. Late-life depression and risk of vascular dementia and Alzheimer's disease: Systematic review and meta-analysis of community-based cohort studies. $\mathrm{Br} \mathrm{J}$ Psychiatry 2013; 202 (5): 329-35.

21. Byers AL, Yaffe K. Depression and risk of developing dementia. Nat Rev Neurol 2011; 7 (6): 323-31.

22. Sacuiu S, Insel PS, Mueller S, Tosun D, Mattsson N, Jack CR Jr, et al. Chronic Depressive Symptomatology in Mild Cognitive Impairment Is Associated with Frontal Atrophy Rate which Hastens Conversion to Alzheimer Dementia. Am J Geriatr Psychiatry 2016; 24 (2): 126-35.

23. McDermott LM, Ebmeier KP. A meta-analysis of depression severity and cognitive function. J Affect Disord 2009; 119 (1-3): 1-8.

24. O'Shea DM, Fieo RA, Hamilton JL, Zahodne LB, Manly JJ, Stern Y. Examining the association between late-life depressive symptoms, cognitive function, and brain volumes in the context of cognitive reserve. Int J Geriatr Psychiatry 2015; 30 (6): 614-22.

25. Opdebeeck C, Martyr A, Clare L. Cognitive reserve and cognitive function in healthy older people: a meta-analysis. Aging, Neuropsychol Cogn 2016; 23 (1): 40-60.

26. Zahodne LB, Tremont G. Unique effects of apathy and depression signs on cognition and function in amnestic mild cognitive impairment. Int J Geriatr Psychiatry 2013; 28 (1): 50-6.

27. Eagle AL, Fitzpatrick CJ, Perrine SA. Single prolonged stress impairs social and object novelty recognition in rats. Behav Brain Res 2013; 256: 591-7.

28. Rock PL, Roiser JP, Riedel WJ, Blackwell AD. Cognitive impairment in depression: a systematic review and meta-analysis. Psychol Med 2014; 44 (10): 2029-40.

29. Campbell NL, Unverzagt F, LaMantia MA, Khan BA, Boustani MA. Risk Factors for the Progression of Mild Cognitive Impairment to Dementia. Clin Geriatr Med 2013; 29 (4): 873-93.

30. Deckers K, van Boxtel MPJ, Schiepers OJ, de Vugt M, Muñoz Sánchez JL, Anstey KJ, et al. Target risk factors for dementia prevention: a systematic review and Delphi consensus study on the evidence from observational studies. Int J Geriatr Psychiatry 2015; 30 (3): 234-46.

31. Vivaldi F, Barra E. Bienestar Psicológico, Apoyo Social Percibido y Percepción de Salud en Adultos Mayores. Ter psicológica 2012; 30 (2): 23-9.

32. Gow AJ, Corley J, Starr JM, Deary IJ. Which social network or support factors are associated with cognitive abilities in old age? Gerontology 2014; 59 (5): 454-63.

33. Sims RC, Hosey M, Levy S-A, Whitfield KE, Katzel LI, 
Waldstein SR. Distinct Functions of Social Support and Cognitive Function Among Older Adults. Exp Aging Res 2014; 40 (1): 40-59.

34. Pillemer S, Holtzer R. The differential relationships of dimensions of perceived social support with cognitive function among older adults. Aging Ment Health 2016; 20 (7): 727-35.

35. Matias-Guiu JA, Fernández de Bobadilla R, Escudero G, Pérez-Pérez J, Cortés A, Morenas-Rodríguez E, et al. Validación de la versión española del test Addenbrooke's Cognitive Examination III para el diagnóstico de demencia. Neurología 2015; 30 (9): 545-51.

36. Yesavage JA, Brink TL, Rose TL, Lum O, Huang V, Adey $\mathrm{M}$, et al. Development and validation of a geriatric depression screening scale: A preliminary report. J Psychiatr Res 1982; 17 (1): 37-49.

37. Mitchell PH, Powell L, Blumenthal J, Norten J, Ironson G, Pitula CR, et al. A short social support measure for patients recovering from myocardial infarction: The ENRICHD Social Support Inventory. J Cardiopulm Rehabil Prev 2003; 23 (6): 398-403.

38. Roldán Tapia L, León I, García J. Construcción de la escala de reserva cognitiva en población española: estudio piloto. Rev Neurol 2011; 52 (11): 653-60.

39. Ullman J, Bentler P. Structural equation modeling. In: Handbook of Psychology 2013; 661-90.

40. Alosco ML, Spitznagel MB, Raz N, Cohen R, Sweet LH, van Dulmen $\mathrm{M}$, et al. Cognitive reserve moderates the association between heart failure and cognitive impair- ment. J Clin Exp Neuropsychol 2012 ; 34 (1): 1-10.

41. Chen Y, Hicks A, While AE. Loneliness and social support of older people in China: a systematic literature review. Health Soc Care Community 2014; 22 (2): 11323.

42. Farias ST, Mungas D, Hinton L, Haan MN. Demographic, neuropsychological, and functional predictors of rate of longitudinal cognitive decline in Hispanic older adults. Am J Geriatr Psychiatry 2011; 19 (5): 440-50.

43. Petersen RC, Smith GE, Waring SC, Ivnik RJ, Kokmen E, Tangelos EG. Aging, Memory, and Mild Cognitive Impairment. Int Psychogeriatrics 1997; 9 (S1): 65-9.

44. Brewster PWH, Melrose RJ, Marquine MJ, Johnson JK, Napoles A, MacKay-Brandt A, et al. Life experience and demographic influences on cognitive function in older adults. Neuropsychology 2014; 28 (6): 846-58.

45. Naqvi R, Liberman D, Rosenberg J, Alston J, Straus S. Preventing cognitive decline in healthy older adults. Can Med Assoc J 2013; 185 (10): 881-5.

46. Adler NE, Snibbe AC. The Role of Psychosocial Processes in Explaining the Gradient Between Socioeconomic Status and Health. Curr Dir Psychol Sci 2003; 12 (4): 119-23.

47. Mielke MM, Vemuri P, Rocca WA. Clinical epidemiology of Alzheimer's disease: assessing sex and gender differences. Clin Epidemiol 2014 ; 6: 37-48.

48. Laws KR, Irvine K, Gale TM. Sex differences in cognitive impairment in Alzheimer's disease. World J Psychiatry 2016; 6 (1): 54-65. 